\title{
WILEY-VCH
}

\section{Quantification of Photophysical Processes in All-polymer Bulk Heterojunction Solar}

Cells

Ahmed H. Balawi, Zhipeng Kan, Julien Gorenflot, * Paola Guarracino, Neha Chaturvedi, Alberto Privitera, Shengjian Liu, Yajun Gao, Lorenzo Franco, Pierre Beaujuge, and Frédéric Laquai* $^{*}$

Dr. A. H. Balawi, Dr. Z. Kan, Dr. J. Gorenflot, Dr. N. Chaturvedi, Dr. S. Liu, Dr. Y. Gao, Prof. P. Beaujuge, Prof. F. Laquai

Materials Science and Engineering Program (MSE), Physical Sciences and Engineering Division (PSE), KAUST Solar Center (KSC), King Abdullah University of Science and Technology (KAUST), Thuwal 23955-6900, Kingdom of Saudi Arabia.

E-mail: frederic.laquai@kaust.edu.sa; julien.gorenflot@kaust.edu.sa

Dr. P. Guarracino, Dr. A. Privitera, Prof. L. Franco

Department of Chemical Sciences, University of Padua, via Marzolo 1, 35131 Padua, Italy.

Prof. Z. Kan

Chongqing Institute of Green and Intelligent Technology, Chinese Academy of Sciences, Chongqing 400714, China.

Prof. S. Liu

School of Chemistry Guangzhou Key Laboratory of Materials for Energy Conversion and Storage, Guangdong Provincial Engineering Technology Research Center for Materials for Energy Conversion and Storage, South China Normal University, Guanghzou, 510006, P. R. China.

Keywords: all-polymer solar cells, organic photovoltaics, non-fullerene acceptors, transient absorption, bulk heterojunction

All-polymer solar cells lack behind the state-of-the-art in small molecule non-fullerene acceptor (NFA) bulk heterojunction (BHJ) organic solar cells (OSCs) for reasons still unclear. In this work, the efficiency-limiting processes in all-polymer solar cells are investigated using blends of the common donor polymer PBDT-TS1 with different acceptor polymers, namely P2TPD[2F]T and P2TPDBT[2F]T. Combining data from steady-state optical spectroscopy and time-resolved photoluminescence (TRPL), transient absorption (TA), and time-delayed collection field (TDCF) experiments, provides not only a concise but also quantitative assessment of the losses due to limited photon absorption, geminate and non-geminate charge carrier recombination, field-dependent charge generation, and inefficient carrier extraction. While both systems exhibit similar charge separation efficiency in the absence of external 


\section{WILEY-VCH}

bias, charge separation is significantly enhanced in P2TPDBT[2F]T-based blends when biased. Kinetic parameters obtained by pulsed laser spectroscopy are used to reproduce the experimentally-measured device JV characteristics and indicate that low fill factors originate either from non-geminate recombination competing with charge extraction, or from a pronounced field dependence of charge generation, depending on the acceptor polymer. The methodology presented here is generic and can be used to quantify the loss processes in BHJ OSCs including both all-polymer and small molecule NFA systems. (196 words)

\section{Introduction}

Organic photovoltaics (OPVs) are an emerging technology with distinct advantages compared to first and second generation solar cell materials specifically, solutionprocessing, semi-transparency, lightweight, and flexibility. ${ }^{[1]}$ Recently, non-fullerenebased devices including all-polymer solar cells have gained remarkable attention and meanwhile have outperformed fullerene-based bulk-heterojunction (BHJ) solar cells. ${ }^{[2]}$ Small molecule non-fullerene acceptors in single-junction OPV devices have demonstrated record power conversion efficiencies (PCE) of up to $18 \%,{ }^{[3]}$ yet allpolymer devices still lag behind with $10-11 \%$ efficiency at best. ${ }^{[4]}$ The main advantages of using non-fullerene acceptors including small molecules and $\pi$-conjugated polymers as the active layer are: (i) complementary absorption spectra and possibility of charge transfer from both components, (ii) tunable energy levels, (iii) balanced charge carrier mobility, and, in particular for all-polymer blends, (iv) improved morphological stability of the donor-acceptor charge percolation networks. ${ }^{[5]}$ The PCE of all-polymer OPV devices increased significantly in the past decade. ${ }^{[6]}$ Numerous studies explored design principles for polymer acceptors including different electron-accepting building blocks such as naphthalene diimide (NDI), perylenediimide (PDI), bithiophene imide (BTI), 


\section{WILEY-VCH}

isoindigo (IID(CO)), and thienopyrroledione (TPD), ${ }^{[c, 7]}$ while other works focused on side chain engineering to control polymer packing in order to improve the blend morphology ${ }^{[8]}$ Moreover, different thin film processing conditions and post-processing treatments such as additives and solvent-vapor annealing (SVA) have been used to improve the device performance. ${ }^{[9]}$ In spite of the efforts, the performance could not match that of small molecule acceptors for still unclear reasons.

An early work by Hodgkiss et al. ${ }^{[10]}$ reported the (photo)physics of all-polymer devices that incorporated F8TBT as the polymer acceptor. This work revealed that subnanosecond geminate charge recombination is a serious loss mechanism in all-polymer blends, unlike many polymer:fullerene systems. Subsequent studies investigated the charge generation in N2200-based polymer acceptor solar cells and reached to the same conclusions, geminate recombination of charge carriers limits the quantum efficiency, for instance in P3HT:N2200 devices. ${ }^{[1]}$ Replacing P3HT by other donor polymers in blends with N2200 was shown to enhance the hole transfer efficiency, while thermal annealing has been used to alter the morphology, which eventually reduced geminate recombination. ${ }^{[12]}$ Recently, Wang et al. reported that photoexcitation of N2200 generates 'intra-moiety polaron pairs' (iPPs) when excitons dissociate and that these iPPs facilitate ultrafast hole transfer at the J51:N2200 interface. ${ }^{[13]}$ To date, most of the spectroscopic studies on all-polymer blends use N2200-based systems, while the effects of different electron-accepting building blocks in the polymer backbone are less well understood. In contrast, fullerene-based systems have been extensively studied and their charge generation and recombination have been quantified, ${ }^{[14]}$ while comprehensive spectroscopic studies of the (photo)physical processes in all-polymer OPVs are still scarce.

Here, we present a systematic study that quantifies loss processes in all-polymer OPV devices, spanning from photon absorption to charge carrier extraction. We performed 


\section{WILEY-VCH}

sub-picosecond to microsecond time-resolved spectroscopy including time-resolved photoluminescence (TRPL), transient absorption (TA), and time-delayed collection field (TDCF) experiments combined with advanced deconvolution of excited state spectra and dynamics to extract accurate exciton dynamics and charge carrier recombination parameters. The (photo)physical parameters were used to simulate the device JV characteristics, confirming the performance differences and the general applicability of our approach to all-polymer solar cells. More specifically, we applied the approach to quantify the (photo)physical losses of a new class of polymer acceptors incorporating thieno[3,4-c]pyrrole-4,6-dione (TPD) and 3,4-difluorothiophene ([2F]T) motifs with branched alkyl-chains. These P2TPD[2F]T and P2TPDBT[2F]T polymers blended with the commercial low-bandgap polymer donor PBDT-TS1 reach device efficiencies of up to $4.4 \%$. We unravel the effect of incorporating the electron-deficient 2,1,3benzothiadiazole (BT) building block into the polymer backbone on the yield of individual (photo)physical processes.

\section{Results and Discussion}

\subsection{BHJ Solar Cell Characterization}

We studied two polymer acceptors, known as P2TPD[2F]T and P2TPDBT[2F]T, whose structures are depicted in Figure 1, in blends with the low-bandgap donor polymer PBDT-TS1. The two analogous polymer acceptors use 2-hexyldecyl (2HD) branched alkyl side chains attached to TPD, resulting in similar solubility in common organic solvents. The difference between the two polymers is the additional benzothiadiazole (BT) moiety in P2TPDBT[2F]T. The synthesis of the acceptor polymers, as well as their performance in BHJ OPV devices, thin film morphology, and charge transport properties 


\section{WILEY-VCH}

have previously been reported by Liu et al. ${ }^{[7 \mathrm{e}]}$ Briefly, the thin film morphology of the two blends is comparable with only slight differences in the nanoscale aggregation. However, the electron mobility in $\mathrm{P} 2 \mathrm{TPD}[2 \mathrm{~F}] \mathrm{T}$-based blends is two orders less than the mobility in P2TPDBT[2F]T-based blends, indicating a potential charge extraction problem, supported also by this study (vide infra).

BT causes a minor change of the energetics of the acceptor polymer however, a significant change of the BHJ device performance is observed. Figure $1 \mathrm{~b}$ depicts the ionization energy (IE) and optical bandgap of the polymers. IEs were determined by photoelectron spectroscopy in air (PESA), while optical bandgaps were estimated at the intersection of the absorption and emission spectra (Figure S1). The presence of BT has little impact on IE, while the optical bandgap decreases, as indicated by a $60 \mathrm{~nm}$ red-shift of the absorption spectrum of P2TPDBT[2F]T (Figure S1). The IE and estimated electron affinity (EA) offsets between the donor and acceptor polymers appear to be sufficient for charge transfer at the D/A interface. The J-V characteristics of optimized solar cells prepared as described earlier ${ }^{[7 e]}$ are presented in Figure 1c. Solar cells with P2TPDBT[2F]T as acceptor exhibit an average PCE of 4.4\%, while P2TPD $[2 \mathrm{~F}] \mathrm{T}$-based devices reach only $1.9 \%$. The PV performance parameters of both all-polymer BHJs in devices are shown in Table 1. Clearly, a substantial increase in $\mathrm{J}_{\mathrm{SC}}$ is observed when using the BT-containing acceptor polymer. 
a)
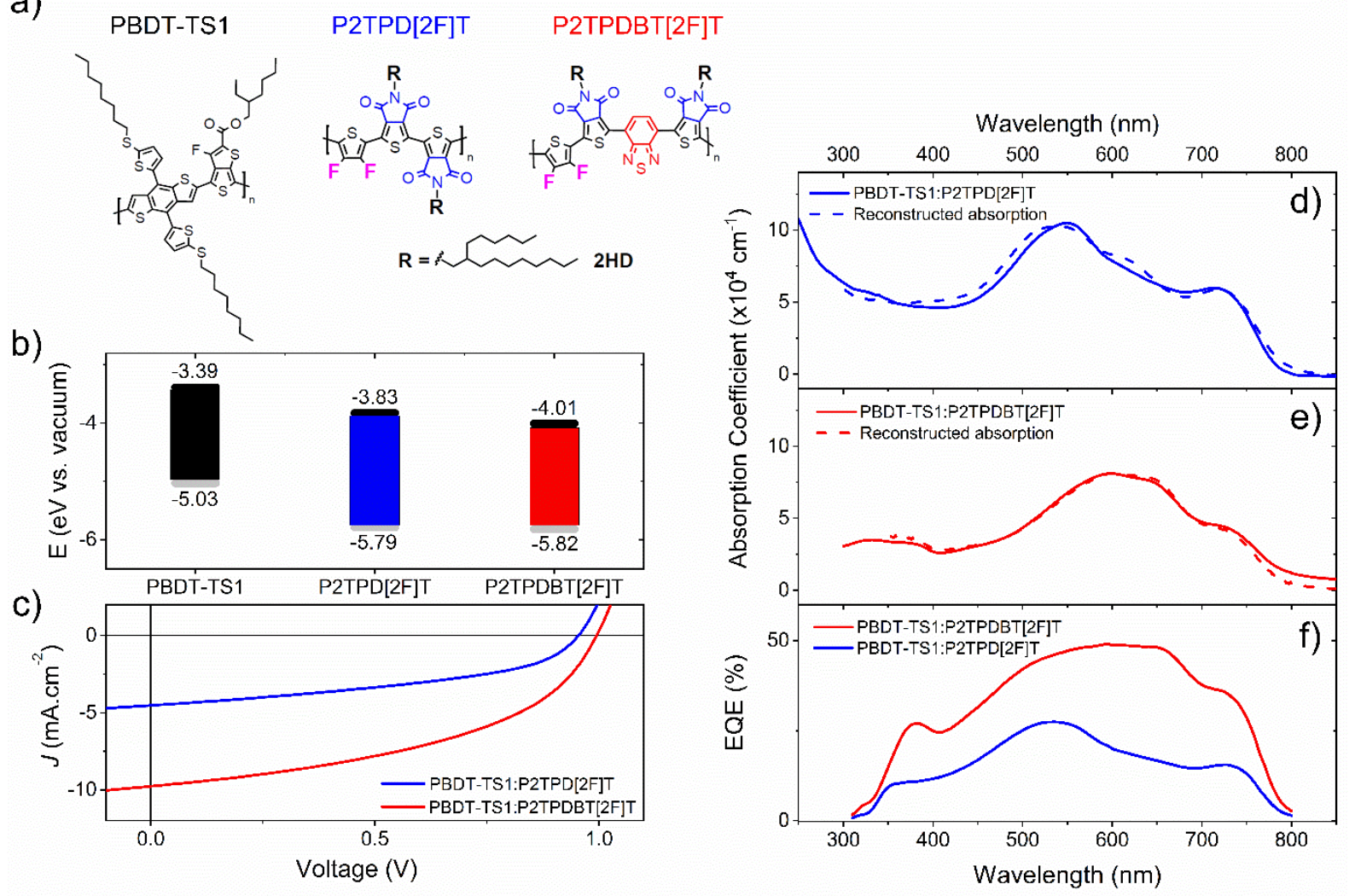

Figure 1. a) Chemical structures of the donor polymer PBDT-TS1 and the acceptor polymers P2TPD[2F]T and P2TPDBT[2F]T. b) Ionization energy (IE) determined by PESA, and electron affinity (EA) estimate based on optical bandgap determined at the intersection of UV-Vis absorbance and PL spectra. c) J-V characteristics of optimized P2TPD[2F]T (blue line) and P2TPDBT[2F]T (red line) BHJ devices. Measured absorption coefficient (solid lines) and reconstructed absorption spectra (dashed lines) for a blend ratio of (1:2) for $\mathrm{d}$ ) PBDT-TS1:P2TPD[2F]T blend (blue lines) with an active layer thickness of $48 \mathrm{~nm}$ and e) PBDT-TS1:P2TPDBT[2F]T blend (red lines) with an active layer thickness of $85 \mathrm{~nm} . \mathrm{f}$ ) EQE spectra of optimized devices.

Table 1. PV performance parameters of BHJs of PBDT-TS1 blended with P2TPD[2F]T or P2TPDBT[2F]T. Optimized BHJ devices had a 1:2 (wt./wt.) ratio and were spin-cast from chloroform (CF) and thermally annealed at $80^{\circ} \mathrm{C}$ for $10 \mathrm{~min}$.

\begin{tabular}{ccccc}
\hline $\begin{array}{l}\text { Polymer } \\
\text { Acceptor }\end{array}$ & $\begin{array}{c}V_{\mathrm{oc}} \\
{[\mathrm{V}]}\end{array}$ & $\begin{array}{c}\mathrm{J}_{\mathrm{sc}} \\
{[\mathrm{mA} / \mathrm{cm} 2]}\end{array}$ & $\begin{array}{c}\mathrm{FF} \\
{[\%]}\end{array}$ & $\begin{array}{c}\text { Avg. PCE } \\
{[\%]^{\mathrm{a})}}\end{array}$ \\
\hline P2TPD[2F]T & $0.96 \pm 0.01$ & $4.6 \pm 0.1$ & $41 \pm 1.4$ & $1.9 \pm 0.1$ \\
P2TPDBT[2F]T & $0.99 \pm 0.00$ & $9.7 \pm 0.2$ & $45 \pm 0.7$ & $4.4 \pm 0.1$ \\
\hline
\end{tabular}

${ }^{\mathrm{a}}$ These values are averages of 10 devices. Device area: $0.1 \mathrm{~cm}^{2}$.

Interestingly, the absorption coefficient of BHJs using the BT-based acceptor is reduced by $22 \%$ compared to $\mathrm{P} 2 \mathrm{TPD}[2 \mathrm{~F}] \mathrm{T}$-based devices, while the incident photon-to-current conversion efficiency (IPCE/EQE) is more than doubled. Figure 1d and 1e show the absorption coefficient of the two blends with maxima of $\sim 8 \times 10^{4} \mathrm{~cm}^{-1}$ at $600 \mathrm{~nm}$ and 


\section{WILEY-VCH}

$10^{5} \mathrm{~cm}^{-1}$ at $550 \mathrm{~nm}$ for P2TPDBT[2F]T and P2TPD[2F]T blends, respectively. The absorption spectra of the blends are a superposition of the absorption spectra of the neat polymers as indicated by the absorption spectra reconstructed (dashed lines) from the neat polymer absorption for a ratio of 1:2 (wt./wt.). The shape of the blends' absorption spectra matches the external quantum efficiency (EQE) spectra (Figure 1f), covering the entire visible spectral region as expected due to the complementary absorption spectra of the neat TPD/[2F]T-based polymer acceptors and the PBDT-TS1 polymer donor. The EQE spectra are dominated by the polymer acceptor contribution and an obvious enhancement in case of the BT-containing acceptor polymer is observed. Interestingly, the P2TPDBT $[2 \mathrm{~F}] \mathrm{T}$ blend exhibits lower absorption coefficients, yet its incident photonto-current conversion efficiency (EQE) is much higher compared to $\mathrm{P} 2 \mathrm{TPD}[2 \mathrm{~F}] \mathrm{T}$ blends. In fact, the low thickness and thus limited absorptance of P2TPD[2F]T-based thin film blends limits their EQE. The thin active layer also affects the balance between charge recombination and extraction, as revealed by transient spectroscopy (vide infra).

\subsection{Exciton Quenching}

We performed TRPL measurements on neat and BHJ blend films, following excitation of the polymer acceptor at $532 \mathrm{~nm}$ (Figure 2). Photoluminescence dynamics were parameterized by bi-exponential fits and weighted-average PL lifetimes of $1.02 \mathrm{~ns}$ for P2TPD[2F]T and 0.28 ns for P2TPDBT[2F]T were determined for the blends (Supplementary Note 2). Quantitative analysis of the blend's PL lifetime compared to the PL lifetime of neat films was performed. Precisely, we calculated the exciton

quenching efficiency as $1-\frac{\tau_{\text {avg,blend }}}{\tau_{\text {avg,neat }}}$. The calculation revealed, PL (exciton) quenching increased from $68.3 \%$ to $86.6 \%$, when using the BT-containing acceptor polymer. 
a)

b)

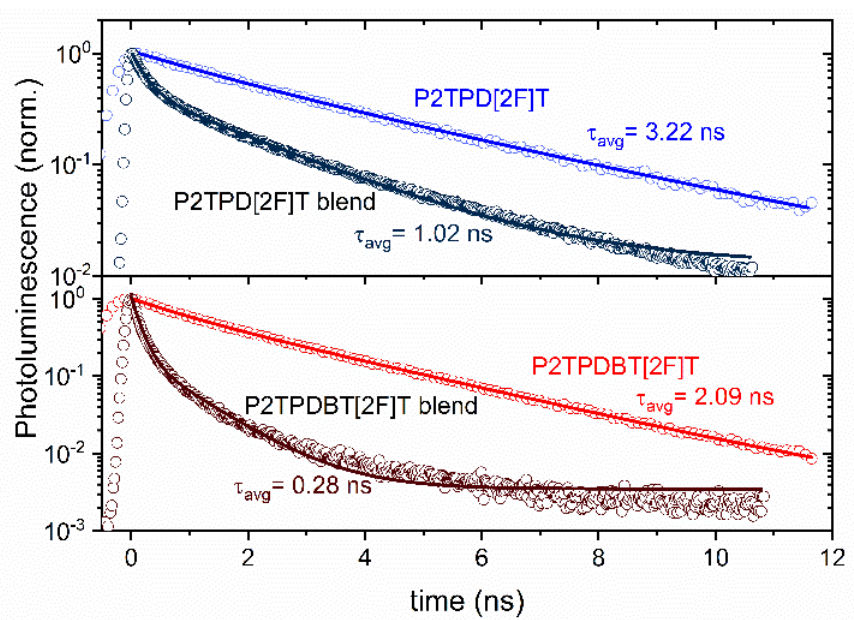

Figure 2. Normalized photoluminescence dynamics of a) neat P2TPD[2F]T films (blue) and PBDT-TS1:P2TPD[2F]T blends (black), b) neat P2TPDBT[2F]T films (light red) and PBDTTS1:P2TPDBT[2F]T blends (black). Solid lines are fits to the data using a bi-exponential decay function. Films were excited at $532 \mathrm{~nm}$ with a fluence of $5.75 \mathrm{~nJ} / \mathrm{cm}^{2}$. Transient PL spectra are shown in Supplementary Note 2.

\subsection{Charge Carrier Generation}

Figure 3 shows transient absorption spectra of the two blends measured in the ns- $\mu \mathrm{s}$ time range following selective excitation of the acceptor polymer. The spectra exhibit ground state bleaching (GSB) above $1.6 \mathrm{eV}$ and excited state absorption (ESA) below $1.6 \mathrm{eV}$. The excited state population of the polymer donor and the polymer acceptor bleach the ground state region at 1.6-1.85 eV and 1.85-2.25 eV, respectively, mimicking the ground state absorption spectra of the materials. Comparing the GSB dynamics it is noteworthy, that the acceptor's GSB signal decays faster than the GSB of the polymer donor. In particular, the GSB of $\mathrm{P} 2 \mathrm{TPD}[2 \mathrm{~F}] \mathrm{T}$ decays to $\sim 50 \%$ of its initial amplitude within 2 ns after photoexcitation, followed by a subtle red-shift. In addition, the ESA features at 0.95 and $1.2 \mathrm{eV}$ exhibit different decays (see Supplementary Note 3), indicating the presence of more than one long-lived state in these blends.

To separate the components contributing to the TA data, multivariate curve resolutionalternating least square (MCR-ALS) analysis was performed yielding the componentassociated spectra and dynamics that contribute to the as-measured TA data. ${ }^{[15]} \mathrm{MCR}-$ 


\section{WILEY-VCH}

ALS analyses revealed two different components in the ns- $\mu$ s time range. The component-associated spectra are shown in Figure $3 \mathrm{c}$ and $3 \mathrm{~d}$, while their dynamics at different excitation fluences are presented in Supplementary Note 3. The spectral signatures of component-1 in either blend (green spectra in Figure 3) resemble the GSB of the polymer donor and acceptor, indicating the presence of charges, that is, donor polymer cations and acceptor polymer anions. The second component (purple spectra) bleaches the polymer acceptor in the case of $\mathrm{P} 2 \mathrm{TPD}[2 \mathrm{~F}] \mathrm{T}$ blends. This component is short-lived (up to ns) and thus assigned to $\mathrm{P} 2 \mathrm{TPD}[2 \mathrm{~F}] \mathrm{T}$ singlet excitons, since its spectral signatures match those observed in the TA of neat P2TPD[2F]T films (Supplementary Note 3) and since its lifetime matches the photoluminescence lifetime of the singlet excitons of about 1 ns (Figure 2b). However, in P2TPDBT[2F]T blends component-2 is much longer-lived, up to hundreds of nanoseconds, and it bleaches only the polymer donor. To reveal the nature of component-2 in the P2TPDBT[2F]T blend, we performed TA on a P2TPDBT[2F]T blend in air. Here, the band observed at probe energies of 0.95 and $1.05 \mathrm{eV}$ decays faster in comparison to measurements done in vacuum (see Supplementary Note 4). Such air-induced quenching is a clear signature of the presence of triplet excitons and thus we assigned component- 2 in P2TPDBT[2F]T blends to triplet states. ${ }^{[16]}$ Monitoring their dynamics (see Figure S4), we observe intensity-dependent triplet formation and recombination in the blend. This indicates that non-geminate recombination of free charges is the origin of triplet state formation, which is in line with previous reports on other OPV systems. ${ }^{[17]}$ 

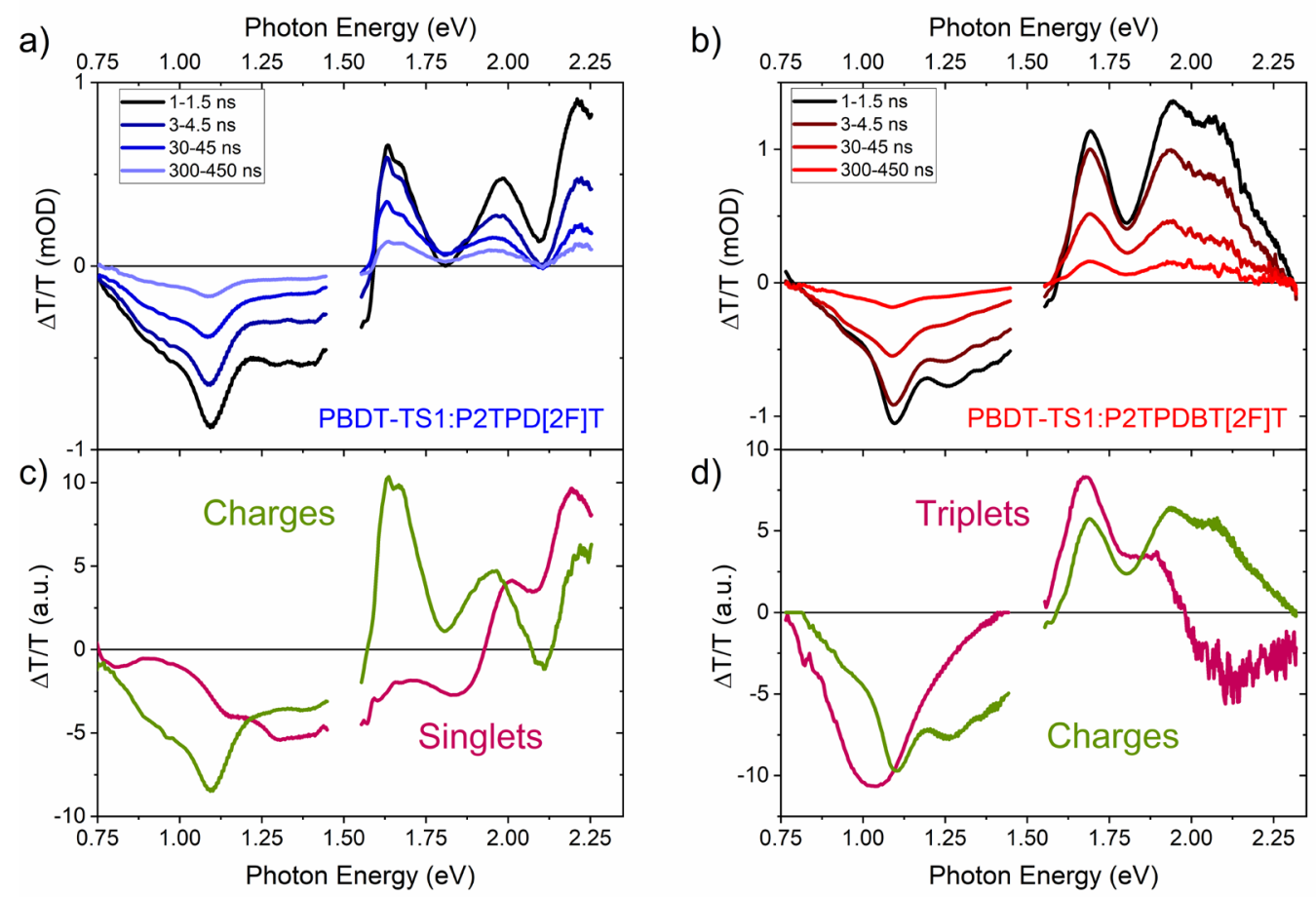

Figure 3. Transient absorption spectra of (a) PBDT-TS 1:P2TPD[2F]T blend (excitation fluence: $8.4 \mu \mathrm{J} / \mathrm{cm}^{2}$ ) and (b) PBDT-TS1:P2TPDBT[2F]T blend (excitation fluence: 2.15 $\mu \mathrm{J} / \mathrm{cm}^{2}$ ). Components of the TA spectra: c) PBDT-TS1:P2TPD[2F]T and d) PBDTTS1:P2TPDBT[2F]T as obtained by MCR-ALS analysis. Films were excited at $532 \mathrm{~nm}$.

To support the spectral assignment shown in Fig. 3d and to reveal in which of the blend's polymers the triplets resided, we probed a pristine PBDT-TS1 film with TRPL and TA (Figure S10). TA spectra at 10-15 ns delay match the triplet-induced spectrum (component-2) obtained by MCR-ALS analysis of the TA data of P2TPDBT[2F]T blends. TA dynamics of the pristine PBDT-TS1 film obtained at different fluences, probing the $0.95-1.05 \mathrm{eV}$ band, indicate that in contrast to blends, triplets in neat PBDTTS1 films are created by intersystem crossing (ISC) from singlet excitons. We also performed TA experiments on neat PBDT-TS1 films in vacuum and in air and when doped with 5 wt.\% platinum octaethylporphyrin (PtOEP), a common triplet sensitizer. The corresponding spectra and dynamics are shown in Figure S11; they demonstrate similar ESA signatures in the $0.95-1.05 \mathrm{eV}$ spectral region as observed in the P2TPDBT $[2 \mathrm{~F}] \mathrm{T}$ polymer blend. Moreover, the triplet-induced signatures were quenched, when the measurement was performed in air, while a weak signal remained 


\section{WILEY-VCH}

that resembled the charge-induced spectra seen also in the blends. These findings altogether support the assignment of the second component in the P2TPDBT[2F]T blend to triplet excitons. Here, triplets are generated by free charge recombination via triplet charge transfer states $\left({ }^{3} \mathrm{CT}\right)$, which subsequently relax to the energetically lowest-lying triplet exciton state $(\mathrm{T})$ present in the blend, that is, the triplet of the donor polymer PBDT-TS1. This process was previously observed for fullerene-based BHJ systems and explained by downhill triplet state transfer from the triplet ${ }^{3} \mathrm{CT}$ state to the lower energy polymer triplet state. ${ }^{[18]}$

To support our findings from TA experiments, we performed Electron Paramagnetic Resonance (EPR) spectroscopy. Time-resolved EPR (TR-EPR) allows the investigation, with a time resolution of about 100 nanoseconds, of the generation and decay dynamics of photo-excited paramagnetic states, such as triplet states and radicals. The TR-EPR of all the polymers show the presence of excited triplet states in the first few microseconds after photoexcitation. The spectra recorded at $5 \mu$ s after excitation and the corresponding zero-field splitting (ZFS) parameters used to simulate the spectra are reported in Supplementary Note 5. In the case of neat PBDT-TS1, the TR-EPR spectral could only be reproduced by including a contribution from charge recombination or back electron transfer (BET) from charge transfer states in addition to the formation of triplet excitons by intersystem crossing (ISC) (see Figure S12). This implies that excited states in the PBDT-TS1 polymer undergo photoinduced charge generation even in the absence of an acceptor, with occasional recombination populating the triplet state of the polymer.

\subsection{Field Dependence of Charge Generation}

We note that both all-polymer blends exhibit moderate fill factors, indicating fielddependent charge generation, a pronounced competition between non-geminate 


\section{WILEY-VCH}

recombination and charge extraction, or either. To reveal a possible field-dependence of charge generation, we used time-delayed collection field (TDCF) measurements, subjecting the devices to pre-bias voltages $\left(\mathrm{V}_{\text {pre }}\right)$ ranging from $-0.5 \mathrm{~V}$ up to $\mathrm{V}_{\mathrm{OC}}$ during the photo-excitation pulse. TDCF measurements were executed at low excitation density $\left(0.1 \mu \mathrm{J} / \mathrm{cm}^{2}\right)$ to reduce the impact of non-geminate recombination prior to charge collection. A collection voltage of $-4 \mathrm{~V}$ was applied $10 \mathrm{~ns}$ after the laser pulse, that is, prior to the expected onset of non-geminate recombination at low fluences. The fluence dependence of the total photo-charge $\left(\mathrm{Q}_{\mathrm{tot}}\right)$ extracted per pulse is shown in Supplementary Note 6. The evolution of $\mathrm{Q}_{\text {tot }}$ as a function of the voltage applied during photoexcitation is shown in Figure 4; here, it is assumed to be solely caused by the increased generation efficiency of separated charges when increasing the internal field from flat band conditions (around $\mathrm{V}_{\mathrm{OC}}$ ) towards larger internal fields. The TDCF measurement on a PBDT-TS1:P2TPD[2F]T device indicated weak field-dependence of free charge generation, seen as a moderate increase of $\mathrm{Q}_{\text {tot }}$ with applied pre-bias. Interestingly, the field-dependence was more pronounced for PBDTTS1:P2TPDBT[2F]T. To quantify the field-dependent losses, we determined the additional loss between open-circuit conditions and a pre-bias of $-0.5 \mathrm{~V}$. On this basis, P2TPD[2F]T devices exhibit $12.8 \%$ (additional) loss due to the field-dependence of charge generation, while $22.5 \%$ are observed for the BT-containing polymer. The impact of the field-dependent losses on the $J-V$ characteristics, especially the fill factor, are indicated in Figure 4 by the shaded areas. However, the results clearly indicate that fielddependence of charge generation cannot be the sole reason for the moderate $F F$ values around $43 \%$. As mentioned, the other possible source of field-dependent photocurrent is a competition between charge extraction and recombination. Thus, we investigated the charge recombination rates in both systems. 


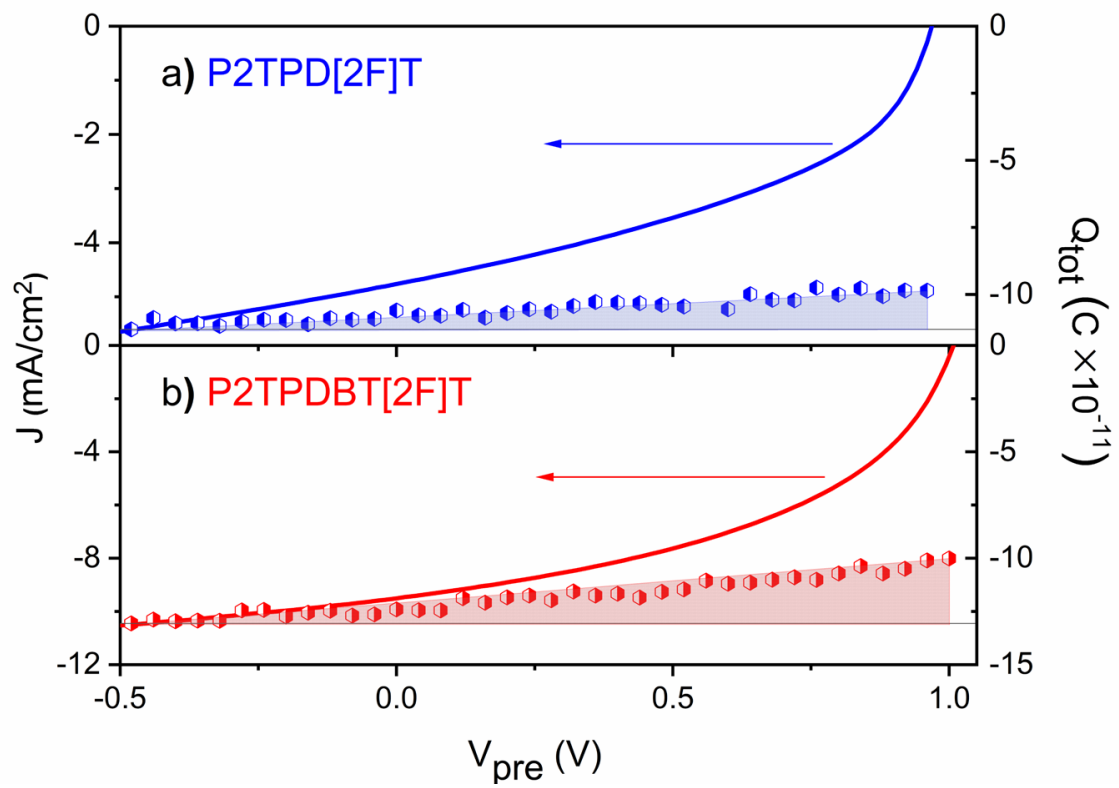

Figure 4. Field dependence of charge generation. J-V characteristics (left axes) and collected charges, $Q_{\text {tot }}$, measured by TDCF for various pre-bias voltages (right axes) for: a) PBDTTS1:P2TPD[2F]T and b) PBDT-TS1:P2TPDBT[2F]T blends. Excitation was performed with a fluence of $0.1 \mu \mathrm{J} / \mathrm{cm}^{2}$ at a wavelength of $532 \mathrm{~nm}$. We note that $Q_{\text {tot }}$ was scaled to match the current at $-0.5 \mathrm{~V}$; shaded areas represent the effect of the field dependence of charge generation.

\subsection{Charge Carrier Recombination}

Parametrizing the transient charge density in both system showed that introducing BT into the polymer backbone has little impact on the fraction geminate charge recombination, but changes the rate of bimolecular recombination. Normalized charge carrier dynamics extracted from MCR-ALS analysis of the TA data of the two systems are shown in Figure 5. The fluence dependent recombination was globally fitted with a previously introduced two-pool charge recombination model. This model assumes charges populate two pools after the initial interfacial charge transfer process. ${ }^{[19]}$ One pool corresponds to separated charges $(f)$ that have overcome their coulombic attraction and in the following recombine non-geminately, while the other pool consists of charges that have failed to overcome the coulombic attraction (1-f) and subsequently recombine geminately at the $\mathrm{D} / \mathrm{A}$ interface. Geminate recombination is independent of the 


\section{WILEY-VCH}

excitation fluence and is characterized by a quasi-exponential decay. The parameters extracted from global fits to the charge carrier dynamics are presented in Table 2. Interestingly, the fraction of geminate recombination is similar in both blends, accounting for $\sim 29 \%$ of the total carrier recombination. However, the spatially-separated (free) charges recombine at very different rates, when comparing the two systems. The 'effective' (bimolecular) recombination rates reveal that incorporation of BT units reduces the rate of bimolecular recombination by one order of magnitude, while the empirical recombination order remains similar $(\sim 2.8)$. This is in line with light-intensity dependent measurements of the $J_{S C}$ of both blends reported earlier by Liu et al., indicating less non-geminate recombination in P2TPDBT[2F]T-based blends. ${ }^{[7 \mathrm{e}]}$ More details of the two-pool recombination model and extraction of recombination parameters are presented in Supplementary Note 7.
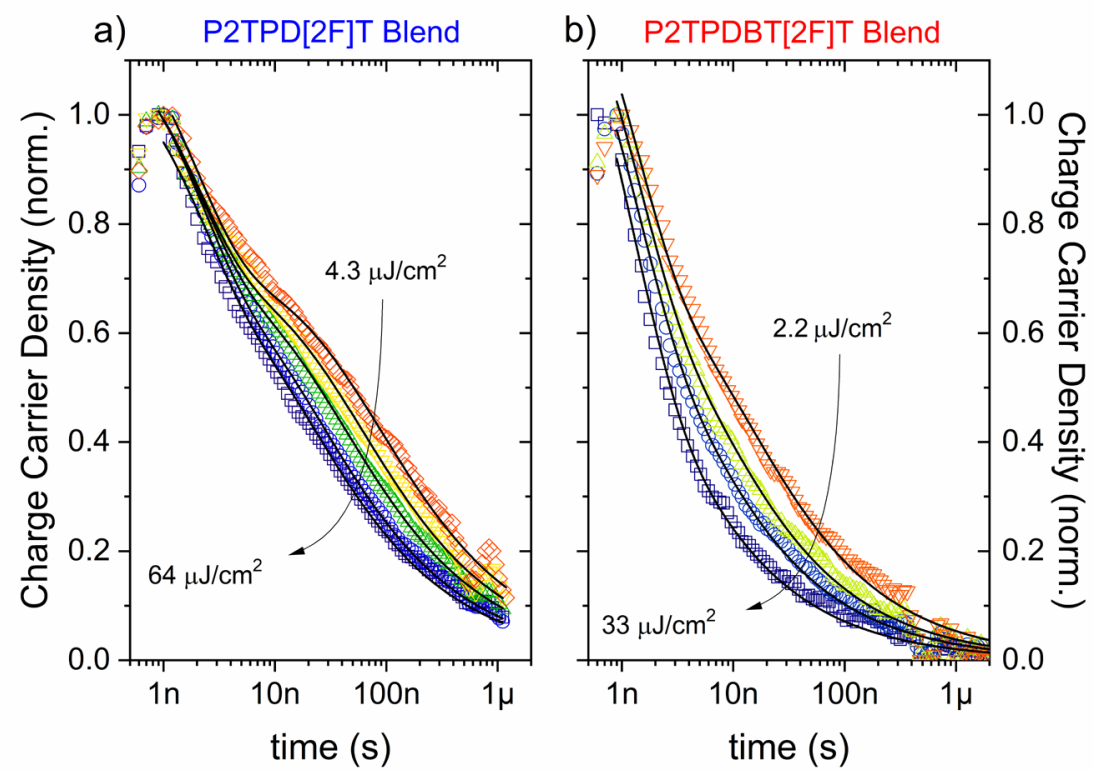

Figure 5. Fluence-dependent charge carrier dynamics of: (a) PBDT-TS1:P2TPD[2F]T and (b) PBDT-TS1:P2TPDBT[2F]T blends photo-excited at $532 \mathrm{~nm}$. Solid black lines are global fits to the fluence-dependent charge recombination dynamics using the two-pool recombination model.

Table 2. Global fit parameters of the two-pool recombination model obtained from the fit to the fluence dependent charge carrier dynamics. The effective bimolecular recombination coefficient was calculated by $k_{2}=k_{\lambda} n_{1 \text { sun }}^{\lambda-1}$, where the carrier density at 1 -sun, $n_{1 \text { sun }}$, was approximated to $1 \times 10^{16} \mathrm{~cm}^{-3}$. 


\begin{tabular}{lccccc}
\hline $\begin{array}{l}\text { Polymer } \\
\text { Acceptor }\end{array}$ & $f$ & $\begin{array}{c}\mathrm{T} \\
{[\mathrm{ns}]}\end{array}$ & $\lambda+1$ & $\begin{array}{c}k_{\lambda} \\
{\left[\mathrm{cm}^{3(\lambda-1)} \mathrm{s}^{-1}\right]}\end{array}$ & $\begin{array}{c}k_{2} \\
{\left[\mathrm{~cm}^{3} \mathrm{~s}^{-1}\right]}\end{array}$ \\
\hline P2TPD[2F]T & 0.72 & 1.92 & 2.9 & $0.00226 n^{-1.375}$ & $1.24 \times 10^{-10}$ \\
P2TPDBT[2F]T & 0.71 & 1.23 & 2.8 & $2.4 \times 10^{-24}$ & $1.5 \times 10^{-11}$ \\
\hline
\end{tabular}

\subsection{Simulation of JV Characteristics}

Lastly, knowing the precise contribution of each loss channel, we carried out simulations of the devices' JV characteristics using SETFOS 5.0 from Fluxim to visualize their impact on a device under solar operating conditions. Here, the charge generation was computed by simulation of the photoactive layer's absorptance in the device stack based on the refractive index determined by spectroscopic ellipsometry (see Supplementary Note 8), taking into account density-independent losses, namely exciton decay and geminate charge recombination. More precisely, the charge generation efficiency (CGE) was calculated as product of the exciton quenching efficiency and the fraction of separated charges (vide supra). As both, exciton quenching and charge separation, were quantified for excitation at $532 \mathrm{~nm}$ only, the CGE was corrected further to account for the pronounced wavelength dependence of the efficiency, as indicated by the IQE spectra, according to: $\mathrm{CGE}_{\mathrm{avg}}=\mathrm{CGE}_{532 \mathrm{~nm}} \times \mathrm{IQE}_{\mathrm{avg}} / \mathrm{IQE}_{532 \mathrm{~nm}}$ (details in Supplementary Note 8). This yielded average CGEs for P2TPD[2F]T and P2TPDBT[2F]T blends of $49.2 \%$ and $61.5 \%$, respectively. As can be seen in Figure 6, the CGE of the $\mathrm{P} 2 \mathrm{TPD}[2 \mathrm{~F}] \mathrm{T}$-based blend is larger than the corresponding IQE, which we assign to extraction losses. We note the IQE spectra exhibit a pronounced wavelength dependence and hypothesize that the generation efficiency depends on whether predominantly the donor or the acceptor is excited, that is, on whether photoinduced electron or hole transfer dominates. The non-geminate recombination rate in the device was determined iteratively from the charge carrier density-dependent non-geminate recombination 


\section{WILEY-VCH}

prefactor, as determined by the two-pool carrier recombination model, and the simulated steady-state charge density distribution across the photoactive layer; see details in Supplementary Note 8 .

The simulated $J-V$ characteristics, precisely the $J_{S C}$ and $V_{O C}$, match the experimental results very well however, significantly larger fill factors, 55\% and 63\%, are obtained from the simulation for P2TPD $[2 \mathrm{~F}] \mathrm{T}$-based and P2TPDBT[2F]T-based devices, respectively (Table 3). Interestingly, the differences in FF (see shaded areas in Figure 6) correspond to the fraction of field-dependent charge generation determined by TDCF measurements (note that the field dependence could not be taken into account in the simulation).
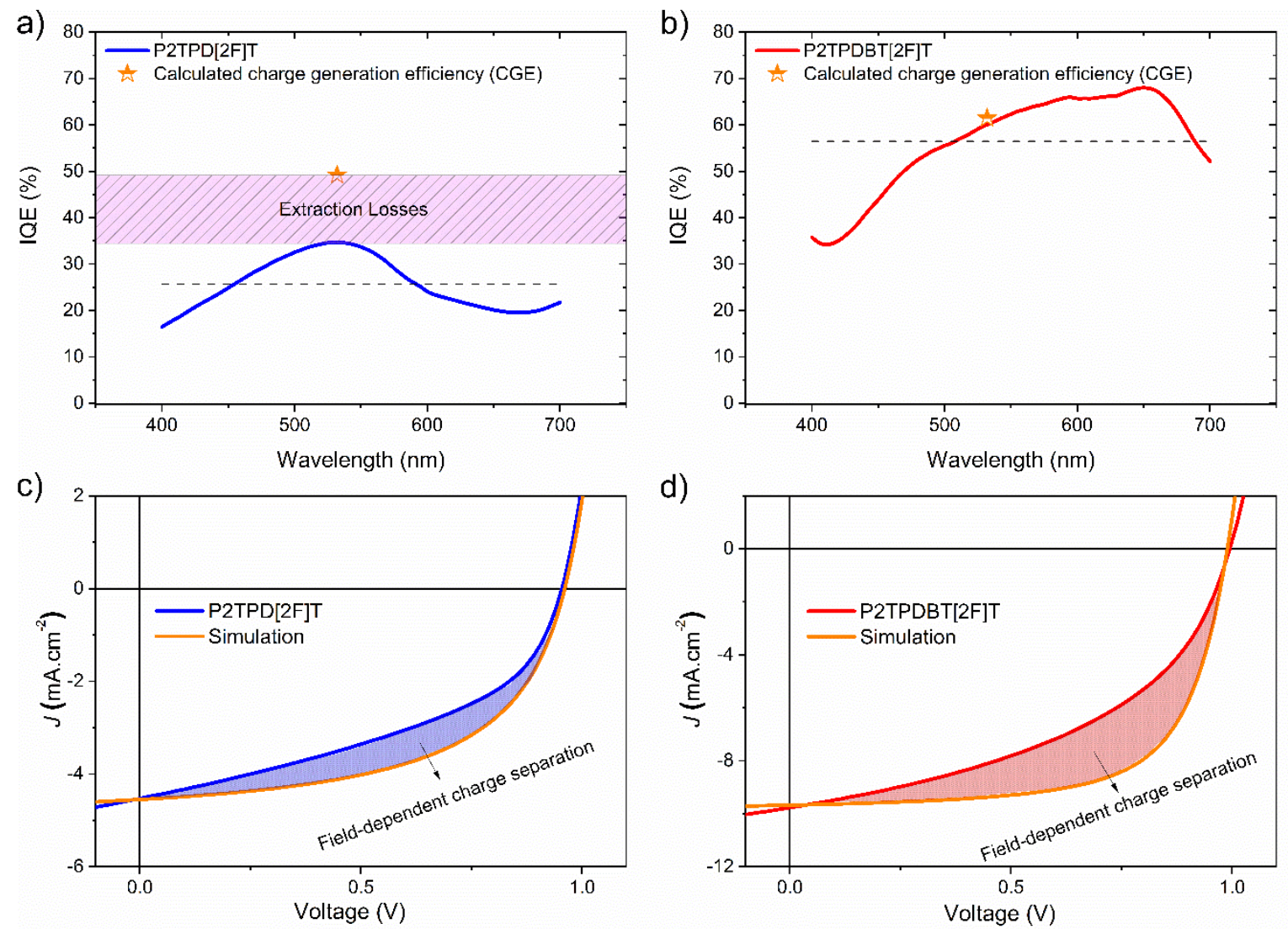

Figure 6. Determination of extraction losses and the effect of field-dependent charge generation on J-V characteristics. Measured IQE spectrum and charge generation efficiency estimated from time-resolved spectroscopic measurements (orange stars) for: a) PBDTTS1:P2TPD[2F]T devices and b) PBDT-TS1:P2TPDBT[2F]T devices. Dashed lines in a) and b) represent the average IQE value determined to scale the calculated charge generation efficiency for SETFOS simulations. Comparison of measured J-V characteristics and simulated J-V characteristics (orange lines) for: c) PBDT-TS1:P2TPD[2F]T devices and d) 


\section{WILEY-VCH}

PBDT-TS1:P2TPDBT[2F]T devices. Shaded areas in c) and d) highlight the losses due to the field-dependence of charge generation, reducing the device fill factor. Orange stars represent the charge generation efficiencies (CGE) calculated from the experimentally-determined kinetic parameters.

Table 3. Device parameters obtained by simulation (using SETFOS) of JV characteristics of all-polymer blends using PBDT-TS1 as donor and P2TPD[2F]T or P2TPDBT[2F]T as acceptor polymer. Differences between the experimentally-measured and simulated parameters are denoted by $\Delta$.

\begin{tabular}{lcccccccc}
\hline $\begin{array}{l}\text { Polymer } \\
\text { Acceptor }\end{array}$ & $\begin{array}{l}\mathrm{V}_{\mathrm{OC}} \\
{[\mathrm{V}]}\end{array}$ & $\begin{array}{c}\Delta_{\mathrm{Voc}} \\
{[\mathrm{V}]}\end{array}$ & $\begin{array}{c}\mathrm{J}_{\mathrm{sc}} \\
{\left[\mathrm{mA} / \mathrm{cm}^{2}\right]}\end{array}$ & $\begin{array}{c}\Delta_{\mathrm{Jsc}} \\
{\left[\mathrm{mA} / \mathrm{cm}^{2}\right]}\end{array}$ & $\begin{array}{c}\mathrm{FF} \\
{[\%]}\end{array}$ & $\begin{array}{c}\Delta_{\mathrm{FF}} \\
{[\%]}\end{array}$ & $\begin{array}{c}\text { Avg. } \\
\mathrm{PCE} \\
{[\%]}\end{array}$ & $\begin{array}{c}\Delta_{\mathrm{PCE}} \\
{[\%]}\end{array}$ \\
\hline P2TPD[2F]T & 0.96 & 0.0 & 4.53 & 0.07 & 55 & 14 & 2.4 & 0.5 \\
P2TPDBT[2F]T & 0.99 & 0.0 & 9.67 & 0.03 & 63 & 18 & 6.2 & 1.8 \\
\hline
\end{tabular}

\subsection{Discussion}

The quantitative spectroscopic analyses of the losses in individual (photo)physical processes provides a concise picture of the origin of the performance differences of the two all-polymer systems reported here. The current after taking into account each loss channel is summarized in Figure 7. Precisely, introducing BT as building block to the acceptor polymer backbone increases the efficiency of exciton quenching by $27 \%$, leading to more efficient charge transfer (CT) state formation. Zero-field efficiency of CT state splitting is similar in both all-polymer blends $(\sim 71 \%)$, as determined from fitting the density dependent ns- $\mu$ s charge carrier dynamics. We note that in both systems the IE offsets should be sufficient for excitons in the acceptor polymer to separate at the $\mathrm{D} / \mathrm{A}$ interface. ${ }^{[20]}$ 


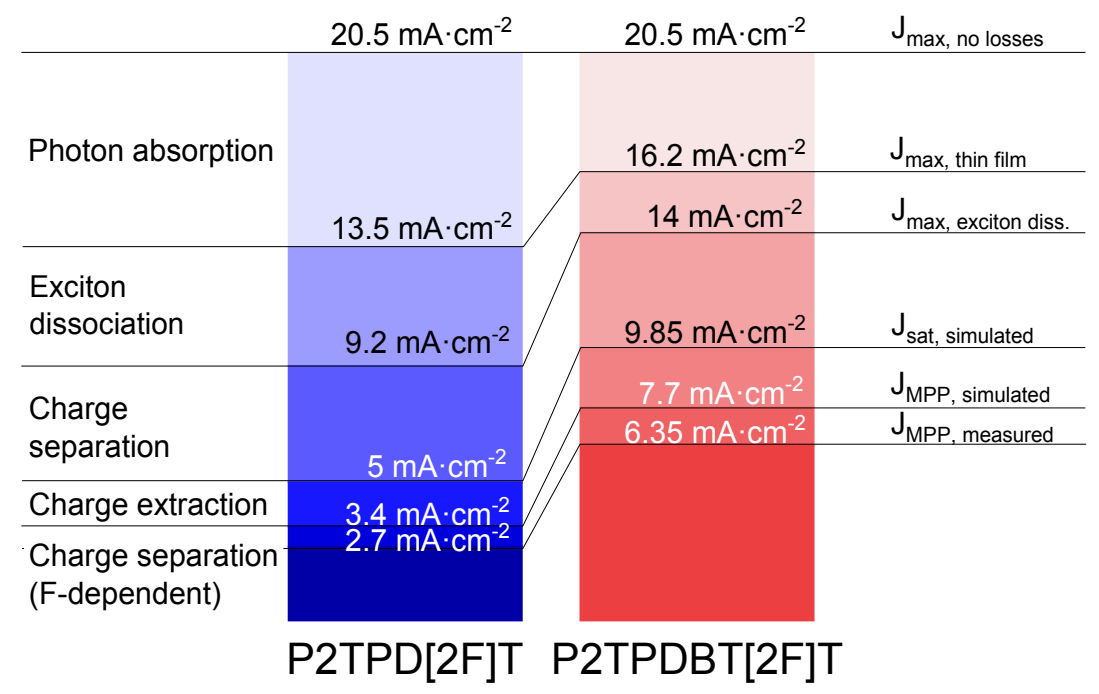

Figure 7. Summary of the photocurrent losses occurring at the maximum power point (at a bias of $0.7 \mathrm{~V}$ for both blends). $J_{\max }$ no losses denotes the calculated maximum achievable photocurrent in the absence of any losses for an active layer thickness of $110 \mathrm{~nm}$ for P2TPD[2F]T-based blends and $135 \mathrm{~nm}$ for P2TPDBT[2F]T-based blends, respectively (see also the thickness-dependent absorption in Figure S19). $J_{\max }$, thin film is the calculated current density expected for an IQE of $100 \%$ and for the experimentally-obtained optimal active layer thickness of $48 \mathrm{~nm}$ for P2TPD[2F]T-based blends and $85 \mathrm{~nm}$ for P2TPDBT[2F]T-based blends, respectively. Furthermore, $J_{\max }$, exciton diss. denotes the photocurrent obtained when including incomplete exciton dissociation, $J_{\text {sat, simulated }}$ is the simulated photocurrent accounting for incomplete charge separation, $J_{M P P}$, simulated denotes the photocurrent at the MPP accounting for extraction losses, and $J_{M P P \text {, measured }}$ denotes the measured photocurrent further reduced by field-dependent charge separation losses.

Strikingly however, the two BHJ systems respond differently to an electric field: charge separation gradually improves with electric field in the P2TPDBT[2F]T-based system, in turn leading to larger $J_{S C}$ but lower $F F$, while the enhancement is only marginal in the P2TPD[2F]T-based system. In fact, the electric field seems to have little effect on charges in $\mathrm{P} 2 \mathrm{TPD}[2 \mathrm{~F}] \mathrm{T}$ as also indicated by its very low electron mobility. Turning to free charges, this low mobility, combined with large recombination rates $(1.24 \times$ $10^{-10} \mathrm{~cm}^{3} \mathrm{~s}^{-1}$ ) results in strong recombination losses in $\mathrm{P} 2 \mathrm{TPD}[2 \mathrm{~F}] \mathrm{T}$-based systems. Drift-diffusion modelling of the JV characteristics revealed that recombination significantly impacts the fill factor and $J_{S C}$ of P2TPD[2F]T-based devices. ${ }^{[21]}$ The extraction losses may be explained by spatially-trapped charges, as recently attributed to 


\section{WILEY-VCH}

dead ends for charge transport in intermixed all-polymer networks. ${ }^{[22]}$ Consequently, the extraction time in $\mathrm{P} 2 \mathrm{TPD}[2 \mathrm{~F}] \mathrm{T}$-based devices has to be minimized by using thin photoactive layers (optimal thickness: $48 \mathrm{~nm}$ ), which despite the large absorption coefficient of P2TPD[2F]T limits significantly the absorptance of the blend and thus the EQE. In contrast, the one order lower bimolecular recombination rate in P2TPDBT[2F]T-based devices shifts the competition between recombination and extraction of free charge carriers towards the latter, resulting in minimal extraction losses as indicated by the higher $F F$ values obtained from simulations.

The excellent match of the experimentally-measured and simulated $J-V$ characteristics emphasizes the impact of the field-dependence of charge generation on the device $F F$ and, to a lesser extent, on $J_{S C}$ and $V_{O C}$. In fact, the parameter used in the simulation that reproduces the $J-V$ characteristics is the fraction of geminate recombination extracted from TA, not TDCF, that is, $29 \%$ vs. $13 \%$ for the P2TPD[2F]T-based blend. Hence, it appears that the field-dependence of charge generation does not correlate with the fraction of geminate recombination, but with the fraction of charges, that require the support of an electric field to separate entirely. This field-dependence accounts for a $2 \%$ total drop in the PCE of P2TPDBT[2F]T-based blends (see Table 3).

Overall, the introduction of BT to the acceptor polymer backbone appears to improve several processes, specifically exciton quenching, field-dependent charge separation, and charge extraction. We hypothesize the origin is improved transport of excitons and charges (electrons). In fact, the DFT calculations reported earlier by Liu et al. ${ }^{[7 e]}$ demonstrated that while both polymers are planar $\left(180^{\circ}\right.$ dihedral angle between successive monomers), P2TPDBT[2F]T exhibits a larger activation energy barrier for torsion along the backbone than $\mathrm{P} 2 \mathrm{TPD}[2 \mathrm{~F}] \mathrm{T}$. We conjecture that this makes the polymer more resilient to backbone torsion, in turn improving its transport properties 


\section{WILEY-VCH}

and most probably also affecting the interfacial donor-acceptor interaction and energetic landscape.

Lastly, the triplet formation from non-geminate recombination does not seem to have a negative impact on the device performance: in fact, we observed less non-geminate recombination losses in the $\mathrm{P} 2 \mathrm{TPDBT}[2 \mathrm{~F}] \mathrm{T}$-based system, which exhibits triplet formation, despite the larger interface, as indicated by the faster exciton quenching in blends. These results are in line with recent work demonstrating that triplet formation from free charge recombination has little effect on device performance under one sun illumination. ${ }^{[23]}$

\section{Conclusion}

In conclusion, the combination of transient spectroscopic measurements allowed us to quantify the individual loss channels in two all-polymer BHJs. Time-resolved PL measurements revealed differences in exciton quenching efficiency, while TA spectroscopy combined with advanced data deconvolution was used to determine the free charge carrier yield and charge carrier recombination rates. The efficiencies and rates were used to model the devices' JV characteristics. A sufficient match of the simulated and the experimentally-determined short circuit current and open-circuit voltage was found, while large differences in simulated and measured fill factors could be explained by field-dependent charge generation as observed by time-delayed collection field experiments.

The combination of spectroscopic studies and device simulation presented here provides a platform that aids identifying the effect of altering the chemical structure and / or using different thin-film processing conditions on the efficiency and rates of individual (photo)physical processes. In the specific case study presented here, we demonstrate that 


\section{WILEY-VCH}

the introduction of benzothiadiazole building blocks to the backbone of the copolymer acceptor leads to changes in the efficiency of various (photo)physical processes occurring in the BHJ and that low fill factors can have very different physical origin, here, strongly field-dependent charge generation vs. non-geminate recombination competing with extraction. Finally, using the methodology reported here, not only allpolymer, but also other OPV blends such as NFA-based systems can be studied and the obtained insight facilitates the design of new materials for photovoltaic applications.

\section{Experimental Section}

Solar cell fabrication: The all-polymer solar cells were prepared on glass substrates with tindoped indium oxide (ITO, $15 \Omega \mathrm{sq}-1$ ) patterned on the surface (device area: $0.1 \mathrm{~cm}^{2}$ ). The $\mathrm{ZnO}$ precursor solution was prepared according to established procedures, ${ }^{[24]}$ and was spincast at 3,000 rpm onto the ITO-coated glass substrates and baked at $150^{\circ} \mathrm{C}$ for $15 \mathrm{~min}$ in air. Immediately after baking the substrates, the samples were then transferred into a dry nitrogen glovebox $\left(<3\right.$ ppm $\left.\mathrm{O}_{2}\right)$ for active layer deposition. All solutions were prepared in the glovebox using the polymers P2TPD[2F]T, P2TPDBT[2F]T as acceptors, and PBDT-TS1 (purchased from Solarmer, $\mathrm{Mw}=36 \mathrm{kDa}$ and $\mathrm{PDI}=2.0$ ) as donor. The polymers were dissolved in chloroform without any solvent additive, and the solutions were stirred overnight at room temperature. Optimized devices were prepared using a PBDTTS1:P2TPD[2F]T (or P2TPDBT[2F]T) ratio of 1:2 (by weight), with an overall concentration of $10 \mathrm{mg} \mathrm{mL}^{-1}$. The active layers were then spin-cast from the solutions in a glove box at $2000 \mathrm{rpm}$ for $30 \mathrm{sec}$, using a programmable spin coater from Specialty Coating Systems (Model G3P-8), resulting in a film of $\sim 85 \mathrm{~nm}$ in thickness. Then the active layer was thermally annealed at $80{ }^{\circ} \mathrm{C}$ for 10 min. Next, the samples were placed in a thermal evaporator for evaporation of a $7 \mathrm{~nm}$ thick 


\section{WILEY-VCH}

molybdenium oxide $\left(\mathrm{MoO}_{3}\right)$ layer evaporated at $0.5 \AA^{-1}$, and a $100 \mathrm{~nm}$ thick layer of silver evaporated at $5 \AA \mathrm{s}^{-1}$, at a pressure of less than $2 \times 10^{-6}$ Torr.

$J-V$ and EQE spectra: J-V measurements were performed in the glovebox with a Leithley 2400 source meter and an Oriel Sol3A Class AAA solar simulator calibrated to 1 sun AM $1.5 \mathrm{G}$ with a KG-5 silicon reference cell certified by Newport. EQE measurements were performed at zero bias by illuminating the device with a dual-grating monochromatic light supplied by xenon arc lamp. The number of incident photons was calibrated for each wavelength by using silicon photodiode by NIST.

TRPL spectroscopy: For time-resolved photoluminescence measurements the samples were excited with the wavelength-tunable output of an Optical Parametric Oscillator (Radiantis Inspire HF-100), itself pumped by the fundamental at $820 \mathrm{~nm}$ of a Ti:sapphire fs oscillator (Spectra Physics MaiTai eHP), yielding 100 fs pulses at a repetition rate of $80 \mathrm{MHz}$. Excitation beam after OPO was routed through pulse picker (PulseSelect, A.P.E) for repetition division. The PL of the samples was collected with an optical telescope consisting of two plano-convex lenses matched in focal length to a spectrograph (PI Spectra Pro SP2300) for wavelength dispersion and detected with a streak camera (Hamamatsu C10910) system for time resolution. The PL data was acquired in photon counting mode using the streak camera software (HPDTA) and exported to Origin Pro 2019 for further analysis. TA spectroscopy: TA spectroscopy measurements were conducted in the time range from 1 nanosecond (ns) to 100 microseconds $(\mu \mathrm{s})$. A fraction of the fundamental output of a titanium:sapphire amplifier, Coherent Legend Duo, operating at $800 \mathrm{~nm}$ with a repetition rate of $3 \mathrm{kHz}$ and energy of $4.5 \mathrm{~mJ}$ was focused into a c-cut $3 \mathrm{~mm}$ thick sapphire window, thereby generating a white-light supercontinuum from 500 to $1600 \mathrm{~nm}$ used as probe pulses. The excitation light (pump pulse) was provided by an actively Q-switched Nd:YVO4 laser (InnoLas picolo AOT) frequency-doubled to provide pulses at $532 \mathrm{~nm}$. The pump laser was 


\section{WILEY-VCH}

triggered by an electronic delay generator (Stanford Research Systems DG535), itself triggered by the transistor-transistor logic sync from the Legend DUO, allowing control of the delay between pump and probe with a jitter of roughly 100 ps. Pump and probe beams were focused to overlap spatially on the sample which was kept under a dynamic vacuum of $<10^{-5}$ mbar. The transmitted fraction of the white light was guided to a custom-made prism spectrograph (Entwicklungsbüro Stresing) where it was dispersed by a prism onto a 512 pixel negative channel metal-oxide-semiconductor linear image sensor (Hamamatsu S8381-512). The probe pulse repetition rate was $3 \mathrm{kHz}$, while the excitation was directly generated at 1.5 $\mathrm{kHz}$ frequency covering a temporal range from $1 \mathrm{~ns}$ to $100 \mu$ s delays, while the detector array was read out at $3 \mathrm{kHz}$. Adjacent diode readings corresponding to the transmission (T) of the sample after excitation and in the absence of an excitation pulse were used to calculate $\Delta \mathrm{T} / \mathrm{T}$. Measurements were averaged over several thousand shots to obtain a good signal-to-noise ratio.

TDCF spectroscopy: The home-built TDCF setup uses the second harmonic (532 nm) of an actively Q-switched sub-ns Nd:YVO4 laser (INNOLAS piccolo AOT) operating at $5 \mathrm{kHz}$ as excitation. To minimize the $\mathrm{RC}$ response time, a small device area of $1 \mathrm{~mm}^{2}$ is used. The samples were measured under dynamic vacuum conditions to avoid any degradation. A Keysight S1160A function generator was used to provide the pre-bias and extraction bias, while a Keysight four channel digital oscilloscope was used to measure the current response of the device.

EPR spectroscopy: EPR experiments were performed on an X-band ELEXSYS E580 Bruker spectrometer, equipped with a dielectric resonator inside an Oxford CF900 cryostat. In Lightinduced EPR experiments, the photoexcitation of the samples was obtained using the white light from a $300 \mathrm{~W}$ Xe lamp. In Time Resolved EPR (TR-EPR) experiments the signal was detected with an instrumental response time of about 100ns. At each magnetic field position 


\section{WILEY-VCH}

the time evolution of EPR signal after pulsed photoexcitation was recorded with a LeCroy fast digital oscilloscope. The photoexcitation was obtained using a Nd:YAG pulsed laser (Quantel Brilliant, $\lambda=532 \mathrm{~nm}$, pulse length $=5 \mathrm{~ns} ; \mathrm{E} /$ pulse $=$ ca. $5 \mathrm{~mJ}$; repetition rate $10 \mathrm{~Hz}$ ). The spectra were acquired averaging 250 transients at each magnetic field point. The spectral simulations were carried out using Easyspin, a Matlab Toolbox. ${ }^{[25]}$

\section{Supporting Information}

Supporting Information is available from the Wiley Online Library or from the author.

\section{Acknowledgements}

This publication is based upon work supported by the King Abdullah University of Science and Technology (KAUST) Office of Sponsored Research (OSR) under Award No: OSR2018-CARF/CCF-3079.

Received: ((will be filled in by the editorial staff))

Revised: ((will be filled in by the editorial staff)) Published online: ((will be filled in by the editorial staff))

\section{References}

[1] a) G. Li, R. Zhu, Y. Yang, Nature photonics 2012, 6, 153; b) O. Inganäs, Adv. Mater. 2018, 30, 1800388; c) S. E. Root, S. Savagatrup, A. D. Printz, D. Rodriquez, D. J. Lipomi, Chem. Rev. 2017, 117, 6467.

[2] a) A. Wadsworth, M. Moser, A. Marks, M. S. Little, N. Gasparini, C. J. Brabec, D. Baran, I. McCulloch, Chem. Soc. Rev. 2019, 48, 1596; b) J. Zhang, H. S. Tan, X. Guo, A. Facchetti, H. Yan, Nat. Energy 2018, 1; c) P. Cheng, G. Li, X. Zhan, Y. Yang, Nat. Photonics 2018, 12, 131.

[3] a) J. Yuan, Y. Zhang, L. Zhou, G. Zhang, H.-L. Yip, T.-K. Lau, X. Lu, C. Zhu, H. Peng, P. A. Johnson, Joule 2019; b) Y. Cui, H. Yao, L. Hong, T. Zhang, Y. Xu, K. Xian, B. Gao, J. Qin, J. Zhang, Z. Wei, Adv. Mater. 2019, 1808356; c) Y. Cui, H. Yao, J. Zhang, T. Zhang, Y. Wang, L. Hong, K. Xian, B. Xu, S. Zhang, J. Peng, Nat. Commun. 2019, 10, 2515; d) Y. Lin, B. Adilbekova, Y. Firdaus, E. Yengel, H. Faber, M. Sajjad, X. Zheng, E. Yarali, A. Seitkhan, O. M. Bakr, Adv. Mater. 2019; e) Y. Lin, Y. Firdaus, M. I. Nugraha, F. Liu, S. Karuthedath, A. H. Emwas, W. Zhang, A. Seitkhan, M. Neophytou, H. Faber, Adv. Sci. 2020, 1903419; f) Q. Liu, Y. Jiang, K. Jin, J. Qin, J. Xu, W. Li, Z. Xiong, K. Sun, S. Yang, X. Zhang, L. Ding, Sci. Bull. 2020, 65, 272.

[4] a) H. Yao, F. Bai, H. Hu, L. Arunagiri, J. Zhang, Y. Chen, H. Yu, S. Chen, T. Liu, J. Y. L. Lai, ACS Energy Lett. 2019, 4, 417; b) Y. Meng, J. Wu, X. Guo, W. Su, L. Zhu, J. Fang, Z.-G. Zhang, F. Liu, M. Zhang, T. P. Russell, Sci. China Chem. 2017, 1; c) J. Wu, Y. Meng, L. Zhu, X. Guo, F. Liu, M. Zhang, J. Mater. Chem. A 2019.

[5] a) H. Kang, W. Lee, J. Oh, T. Kim, C. Lee, B. J. Kim, Acc. Chem, Res. 2016, 49, 2424; b) H. Benten, D. Mori, H. Ohkita, S. Ito, J. Mater. Chem. A 2016, 4, 5340; c) L. 


\section{WILEY-VCH}

Gao, Z. G. Zhang, L. Xue, J. Min, J. Zhang, Z. Wei, Y. Li, Adv. Mater. 2016, 28, 1884.

[6] a) G. Wang, F. S. Melkonyan, A. Facchetti, T. J. Marks, Angew. Chem. International Edition 2019, 58, 4129; b) C. Lee, S. Lee, G.-U. Kim, W. Lee, B. J. Kim, Chem. Rev. 2019.

[7] a) Y. Guo, Y. Li, O. Awartani, H. Han, J. Zhao, H. Ade, H. Yan, D. Zhao, Adv. Mater. 2017, 29, 1700309; b) Y. Guo, Y. Li, O. Awartani, J. Zhao, H. Han, H. Ade, D. Zhao, H. Yan, Adv. Mater. 2016, 28, 8483; c) F. Cruciani, M. Babics, S. Liu, D. Carja, D. Mantione, P. M. Beaujuge, Macromol. Chem. Phys. 2019, 1900029; d) S. Liu, Y. Firdaus, S. Thomas, Z. Kan, F. Cruciani, S. Lopatin, J. L. Bredas, P. M. Beaujuge, Angew. Chem. 2018, 130, 540; e) S. Liu, X. Song, S. Thomas, Z. Kan, F. Cruciani, F. Laquai, J. L. Bredas, P. M. Beaujuge, Adv. Energy Mater. 2017, 7, 1602574; f) Y. Wang, Z. Yan, H. Guo, M. A. Uddin, S. Ling, X. Zhou, H. Su, J. Dai, H. Y. Woo, X. Guo, Angew. Chem. International Edition 2017, 56, 15304; g) K. Zhou, X. Zhou, X. Xu, C. Musumeci, C. Wang, W. Xu, X. Meng, W. Ma, O. Inganäs, Polymers 2019, 11, 1665.

[8] a) C. Lee, H. Kang, W. Lee, T. Kim, K. H. Kim, H. Y. Woo, C. Wang, B. J. Kim, Adv. Mater. 2015, 27, 2466; b) X. Liu, C. Zhang, C. Duan, M. Li, Z. Hu, J. Wang, F. Liu, N. Li, C. J. Brabec, R. A. Janssen, J. Am. Chem. Soc. 2018, 140, 8934.

[9] a) S. Li, H. Zhang, W. Zhao, L. Ye, H. Yao, B. Yang, S. Zhang, J. Hou, Adv. Energy Mater. 2016, 6 (5), 1501991; b) Z. Li, X. Xu, W. Zhang, X. Meng, W. Ma, A. Yartsev, O. Inganas, M. R. Andersson, R. A. Janssen, E. Wang, J. Am. Chem. Soc. 2016, 138, 10935; c) X. Liu, S. Huettner, Z. Rong, M. Sommer, R. H. Friend, $A d v$. Mater. 2012, 24, 669.

[10] J. M. Hodgkiss, A. R. Campbell, R. A. Marsh, A. Rao, S. Albert-Seifried, R. H. Friend, Phys. Rev. Lett. 2010, 104, 177701.

[11] a) M. Schubert, B. A. Collins, H. Mangold, I. A. Howard, W. Schindler, K. Vandewal, S. Roland, J. Behrends, F. Kraffert, R. Steyrleuthner, Z. Chen, K. Fostiropoulos, R. Bittl, A. Salleo, A. Facchetti, F. Laquai, H. W. Ade, D. Neher, Adv. Funct. Mater. 2014, 24, 4068; b) J. R. Moore, S. Albert - Seifried, A. Rao, S. Massip, B. Watts, D. J. Morgan, R. H. Friend, C. R. McNeill, H. Sirringhaus, Adv. Energy Mater. 2011, 1, 230.

[12] a) J. Yuan, W. Guo, Y. Xia, M. J. Ford, F. Jin, D. Liu, H. Zhao, O. Inganäs, G. C. Bazan, W. Ma, Nano Energy 2017, 35, 251; b) Y. J. Hwang, B. A. Courtright, A. S. Ferreira, S. H. Tolbert, S. A. Jenekhe, Adv. Mater. 2015, 27, 4578; c) K. D. Deshmukh, S. K. Prasad, N. Chandrasekaran, A. C. Liu, E. Gann, L. Thomsen, D. Kabra, J. M. Hodgkiss, C. R. McNeill, Chem. Mater. 2017, 29, 804; d) K. D. Deshmukh, T. Qin, J. K. Gallaher, A. C. Liu, E. Gann, K. O'Donnell, L. Thomsen, J. M. Hodgkiss, S. E. Watkins, C. R. McNeill, Energy Environ. Sci. 2015, 8, 332; e) S. Karuthedath, A. Melianas, Z. Kan, V. Pranculis, M. Wohlfahrt, J. I. Khan, J.

Gorenflot, Y. Xia, O. Inganäs, V. Gulbinas, J. Mater. Chem. A 2018, 6, 7428; f) F. Jin, G. Ding, Y. Wang, J. Yuan, W. Guo, H. Yuan, C. Sheng, W. Ma, H. Zhao, J. Phys. Chem. C 2017, 121, 8804; g) F. Jin, J. Yuan, W. Guo, Y. Xu, Y. Zhang, C. Sheng, W. Ma, H. Zhao, Adv. Funct. Mater. 2018, 28, 1801611.

[13] R. Wang, Y. Yao, C. Zhang, Y. Zhang, H. Bin, L. Xue, Z.-G. Zhang, X. Xie, H. Ma, X. Wang, Nat. Commun. 2019, 10, 398.

[14] a) J. Gorenflot, A. Paulke, F. Piersimoni, J. Wolf, Z. Kan, F. Cruciani, A. E. Labban, D. Neher, P. M. Beaujuge, F. Laquai, Adv. Energy Mater. 2018, 8, 1701678; b) O. Alqahtani, M. Babics, J. Gorenflot, V. Savikhin, T. Ferron, A. H. Balawi, A. Paulke, Z. Kan, M. Pope, A. J. Clulow, Adv. Energy Mater. 2018, 8, 1702941. 


\section{WILEY-VCH}

[15] a) I. Howard, H. Mangold, F. Etzold, D. Gehrig, F. Laquai, World Scientific, Singapore, 2013; b) J. Jaumot, R. Gargallo, A. de Juan, R. Tauler, Chemom. Intell. Lab. Syst. 2005, 76, 101.

[16] a) C. Keiderling, S. Dimitrov, J. R. Durrant, J. Phys. Chem. C 2017, 121, 14470; b) Y. W. Soon, H. Cho, J. Low, H. Bronstein, I. McCulloch, J. R. Durrant, ChemComm 2013, 49, 1291.

[17] D. W. Gehrig, I. A. Howard, F. Laquai, J. Phys. Chem. C 2015, 119, 13509.

[18] a) G. Lakhwani, A. Rao, R. H. Friend, Annu. Rev. Phys. Chem 2014, 65, 557; b) A. Rao, P. C. Chow, S. Gélinas, C. W. Schlenker, C.-Z. Li, H.-L. Yip, A. K.-Y. Jen, D. S. Ginger, R. H. Friend, Nature 2013, 500, 435.

[19] I. A. Howard, R. Mauer, M. Meister, F. Laquai, J. Am. Chem. Soc. 2010, 132, 14866.

[20] C. Poelking, D. Andrienko, J. Am. Chem. Soc. 2015, 137, 6320.

[21] R. Mauer, I. A. Howard, F. Laquai, J. Phys. Chem. Lett 2010, 1, 3500.

[22] R. Jasiūnas, A. Melianas, Y. Xia, N. Felekidis, V. Gulbinas, M. Kemerink, $A d v$. Electron. Mater. 2018, 4, 1800144.

[23] S. Karuthedath, J. Gorenflot, Y. Firdaus, W. Y. Sit, F. Eisner, A. Seitkhan, M. K. Ravva, T. D. Anthopoulos, F. Laquai, Adv. Energy Mater. 2019, 9, 1802476; b) S. Karuthedath, J. Gorenflot, A. Melianas, Z. Kan, M. Kemerink, F. Laquai, J. Phys. Chem. Lett. 2020, just accepted.

[24] L. K. Jagadamma, M. Abdelsamie, A. El Labban, E. Aresu, G. O. N. Ndjawa, D. H. Anjum, D. Cha, P. M. Beaujuge, A. Amassian, J. Mater. Chem. A 2014, 2, 13321.

[25] S. Stoll, A. Schweiger, J. Magn. Reson. 2006, 178, 42. 


\section{Quantification of Photophysical Processes in All-polymer Bulk Heterojunction Solar}

Keywords: all-polymer solar cells, organic photovoltaics, non-fullerene acceptors, transient absorption, bulk heterojunction

Ahmed H. Balawi, Zhipeng Kan, Julien Gorenflot, * Paola Guarracino, Neha Chaturvedi, Alberto Privitera, Shengjian Liu, Yajun Gao, Lorenzo Franco, Pierre Beaujuge, and Frédéric Laquai*

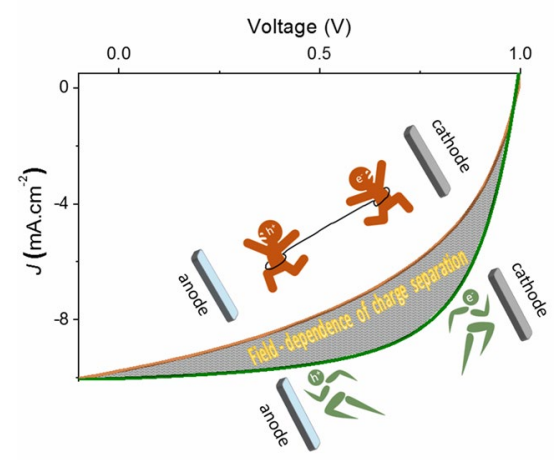

Combined data of transient optical and electro-optical experiments reveals the efficiencydetermining processes in all-polymer solar cells and allows precisely quantifying their yields. For the test system presented here, field-dependent charge separation is shown to limit the fill factor and thus the performance by comparing the experimentally-measured current-voltage characteristics to those reproduced by drift-diffusion simulations using the spectroscopicallydetermined kinetic parameters. 